\title{
Label-free biosensor for viruses and bacteria detection
}

\section{Emir Aznakayev, Diana Aznakayeva}

Emir Aznakayev, Diana Aznakayeva, "Label-free biosensor for viruses and bacteria detection," Proc. SPIE 11378, Nano-, Bio-, Info-Tech Sensors, and 3D Systems IV, 113781G (30 April 2020); doi: 10.1117/12.2572685

SPIE. Event: SPIE Smart Structures + Nondestructive Evaluation, 2020, Online Only 


\title{
Label-free biosensor for viruses and bacteria detection
}

\author{
Emir Aznakayev*a,b, Diana Aznakayeva ${ }^{\mathrm{a}}$, \\ ${ }^{a}$ National Aviation University, prospect Komarova 1, 03058 Kiev, Ukraine; \\ bNational Technical University of Ukraine ‘KPI’, prospect Peremogy 37, 03056 Kiev, Ukraine
}

\begin{abstract}
Construction of graphene-based nanosensor is presented for the detection of viruses and bacteria as infectious agents. Viruses and bacteria are cause of different form of diseases. Detection mechanism for classification and identification of biological agents as well as viruses are paramount at early stages of illness. Fabrication of graphene nanosensor based on surface plasmon resonance (SPR) technique and field-effect for viruses, bacteria, proteins, and nucleic acids detection is proposed in this article.
\end{abstract}

Keywords: biosensor, biosensor construction, virus detection, graphene, device

\section{INTRODUCTION}

The cause of many diseases is the action of viruses, as COVID-19, and bacteria in an organism. The timely detection of viruses and bacteria prevents their harmful influence on an organism. Application of nanocomposites and surface plasmon resonance (SPR) techniques allows quickly and at low cost to perform detection of viruses, bacteria and other biomolecules. There are some papers ${ }^{1,2,3}$ describing such approach for biological objects detection and different biosensors construction.

There is a large group of living organisms that do not have a cellular structure. These organisms are called as viruses and they are non-cellular forms of life. Viruses cannot be carried neither to animals, nor to plants. They are exclusively small and can be studied only by means of an electronic microscope.

Viruses can live and grow only in cells of other organisms. Outside the cells of living organisms, viruses can not survive and many of them in the environment are in the form of crystals. Settled within the cells of animals and plants, the viruses cause many dangerous diseases. Among the virus diseases of plants are known mosaic disease of tobacco, peas and other crops. Each virus particle consists of a small amount of DNA or RNA, that is the genetic material enclosed in a protein shell. This shell has a protective role for a virus.

All viruses common link is that they are autonomous genetic entities which able to operate only in cells with varying degrees depending on the cellular systems, the synthesis of nucleic acids and total dependence on cell protein synthesizes and energy systems, which are amenable to self-evolution. If we look at viruses in terms of parasitology, then their parasites must be recognized not only intracellular (as is the case with rickettsia and chlamydia), and genetic parasitism, as the interaction of the virus with the cell is primarily the interaction of two genomes - a virus and cell.

There were proposed three main hypotheses regarding the origin of viruses. According to the first of them, viruses are descendants of bacteria or other single-celled organisms that suffered degenerative evolution. According to the second, viruses are descendants of ancient pre-cellular life forms that have moved to parasitic mode of existence. As far as the third one, viruses are the result of cellular genetic structures, which have become relatively autonomous, but still retained dependence on the cells. The diversity of genetic material in viruses is one of the arguments about the origin of viruses from pre-cellular life forms. Currently existing viruses are the products of evolution, as their ancient ancestors, and newly emerging autonomous genetic structures.

*aznakayev@nau.edu.ua;www.nau.edu.ua

Nano-, Bio-, Info-Tech Sensors, and 3D Systems IV, edited by Jaehwan Kim, Proc. of SPIE Vol. 11378 $113781 \mathrm{G} \cdot$ @ $2020 \mathrm{SPIE} \cdot \mathrm{CCC}$ code: 0277-786X/20/\$21 · doi: 10.1117/12.2572685 


\section{PROBLEM DESCRIPTION}

Graphene biosensor based on surface plasmon-polariton resonance effect enables real-time, label-free and low-cost detection, identification and classification of viruses, bacteria, biomolecules, proteins and nucleic acids. The twodimensional nature of graphene with its plethora of unique properties such as chemical inertness, biological compatibility, high value of surface to volume ratio, optical and electrical conductivity make it suitable and perfect candidate for the construction of cheap and label-free sensors for biological and medical applications. Furthermore, surface plasmonpolariton resonance technique is extremely surface sensitive method that allows to detect and examine biological species being attached to the surface of graphene-based biosensor. It allows the real-time and label-free detection and analysis due to the interfacial refractive index changes which is associated with binding of biomolecules in the tested solution with biosensor surface.

The design of developed nanosensor comprises of capillary tube, two electrodes, radiation source and photodetector. The first upper transparent electrode is constructed from two-dimensional material graphene. Optical properties of this electrode plate vary according to the number of viruses and biological molecules adsorbed on its surface. Permittivity, reflective index and absorption properties of this electrode depend on its surface structure which varies if bacteria or viruses bonded to the electrode. The electronic and optical characteristics of electrode change as well if bacteria or viruses bonded to electrode. The negative electric charge is located on the surface of bacterial and viral cells. In the case when a positive electric voltage is applied to the electrode plate these biological cells are attracted to the electrode surface. The dielectric layer which consist from biological cells will appear on the bottom side of upper electrode plate. The permittivity of this dielectric layer is defined by the type of absorbed cells. The adsorption rate of biomolecules on the electrode surface is governed by the amount of electrical voltage applied between the electrodes and depends on electrical charge, mass and the type of a virus or biomolecule adsorbed on electrode surface. The accumulation of biological component on electrode surface leads to a change of dielectric permittivity of material on electrode surface and consequently to the measured frequency shift of surface plasmon-polariton resonance (SPPR). From the magnitude of SPPR shift the type of virus or biomolecule deposited on electrode area can be easily identified. The change of dielectric permittivity of medium between two electrodes also alters the total capacitance of a capacitor which is formed by the plates of electrodes, as well as measured electric charges flow in capacitor circuit. Set of these measured parameters allows identify with high accuracy viruses and biomolecules by its types and varieties. It was experimentally presented that the nanoparticle image intensity is proportional linearly to the virus or bacterium cell's dimension. Each virus cell has a stable protein shell and the carrier of a viral genome (viral DNA or RNA) in it and is characterized with a high polarizability.

\section{METHOD for BIOLOGICAL AGENTS DETECTION with GRAPHENE NANOSENSOR}

Graphene nanosensor's scheme is presented in Fig. $1^{4}$ and the virus or bacterium cell motion in the test capillary tube are shown as well: 1 is upper electrode with positive electric potential $(+\varphi) ; 2$ is bottom electrode with negative electric potential $(-\varphi)$; VC is virus or bacterium cell with negative electric potential $\left(-\varphi_{\mathrm{vc}}\right)$ on their shell; $\overrightarrow{\boldsymbol{V}}$ is velocity of virus or bacterium cell in the test capillary tube; $\overrightarrow{\boldsymbol{V}}_{\mathbf{1}}$ is velocity of virus or bacterium cell in the test capillary tube in the normal direction to electrodes in external electric field; $\bar{R}_{0}$ is distance from axis of the test capillary tube to the electrode; $\bar{a}$ is diameter of virus or bacterium cell; $\vec{r}$ is radius-vector normal to the electrode.

The electric voltage $U$ between upper electrode 1 with electric potential $\varphi_{1}$ and bottom electrode 2 with electrical potential $\varphi_{2}$ is presented in Fig. 1

$$
U=\varphi_{1}-\varphi_{2}
$$




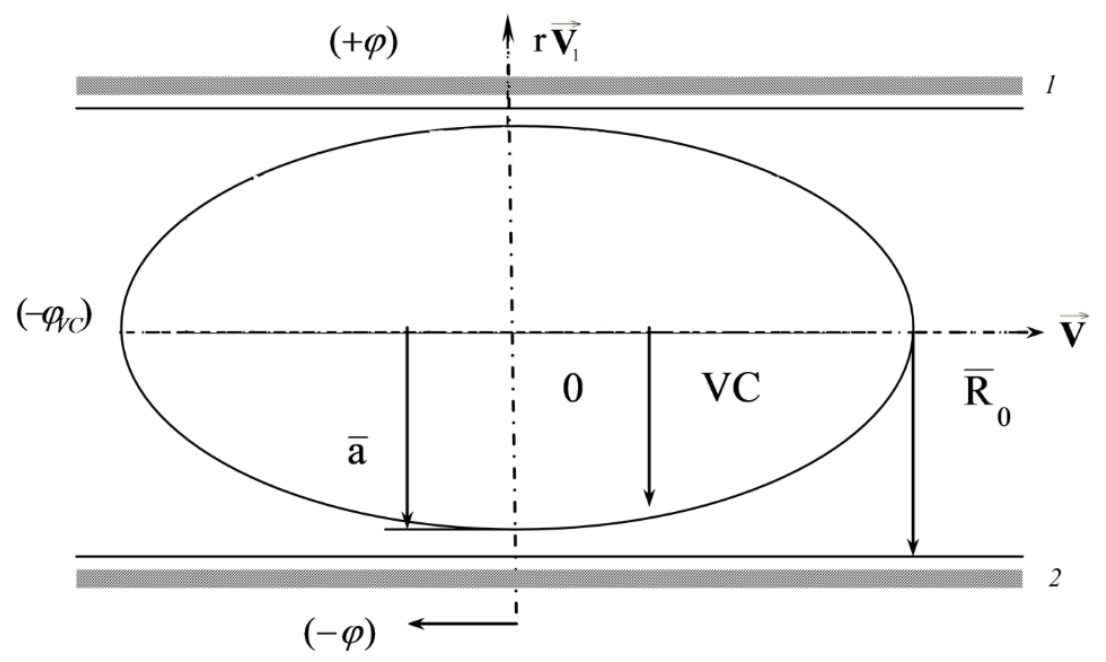

Figure 1. Graphene nanosensor's test tube scheme ${ }^{4}$

The expression for number $N_{v}$ of virus or bacterium cells in the tested substance is ${ }^{4}$

$$
N_{v}=\frac{C_{0} U_{0}}{Q_{v 1}}\left(1-\frac{C_{3}}{C_{0}} \cdot \frac{U_{3}}{U_{0}}\right) .
$$

where $Q_{v 1}$ is the negative electric charge of the unit virus or bacterium cell, $C_{0}$ is capacity of test tube at the absence of virus or bacterium cells, $C_{3}$ is an effective capacity of test tube at the presence of virus or bacterium cells, $U_{0}$ is electric voltage between electrodes 1 and 2 at the absence of virus or bacterium cells; $U_{3}$ is electric voltage between electrodes 1 and 2 at the presence of virus or bacterium cells.

Values in the right part of equation (1) are measurable in experiment, expression for $Q_{v 1}$ is presented below. Thus, we can find the value of $N_{v}$. And the expression for density number of virus or bacterium cells in the tested substance $n_{v}$ is

$$
n_{v}=\frac{\varepsilon_{0} \varepsilon_{2} U_{0}}{Q_{v 1} \cdot d^{2}}\left(1-\frac{C_{3}}{C_{0}} \cdot \frac{U_{3}}{U_{0}}\right),
$$

where $\varepsilon_{0}$ is electric constant; $\varepsilon_{2}$ is permittivity of substance between electrodes at the absence of virus or bacterium cells; $d$ is the distance between electrodes.

To determine the volume per virus or bacterium cell $v_{v 1}$ we assume that the deposition of these cells on the electrode is single-layer (i.e. the concentration of viral or bacterial particles in the substance is moderate). Then the value of thickness $d_{1}$ of virus or bacterium cells layer on upper electrode 1 can be taken equal to the size of one viral or bacterial cell and the expression for the volume $v_{v 1}$ takes the form:

$$
v_{v 1}=4 \pi d_{1}^{3} / 3
$$


The volume of the viral or bacterial cells layer $V_{v}$ on the upper electrode 1 of the test tube equals $V_{v}=N_{v} v_{v 1}$, where $V_{v}=S d_{1}$.

From these expressions with take into account that $n_{v}=N_{v} / V_{0}$, we have expression for $n_{v}$ :

$$
n_{v}=3 S / 4 V_{0} d_{1}^{2}=3 / 4 d d_{1}^{2}
$$

where $V_{0}=S d$ is volume of test tube, $S$ is area of plate of capacitor.

From expressions (2) and (4) we can find expression for electric charge of the unit virus or bacterium cell $Q_{v 1}$ :

$$
3 / 4 d d_{1}^{2}=\varepsilon_{0} \varepsilon_{2} U_{0}\left(1-C_{3} U_{3} / C_{0} U_{0}\right) / Q_{v 1} d^{2}
$$

Hence

$$
Q_{v 1}=4 \varepsilon_{0} \varepsilon_{1} d_{1}^{2} U_{0}\left(1-C_{3} U_{3} / C_{0} U_{0}\right) / 3 d
$$

Values $v_{v 1}$ and $Q_{v 1}$ are characteristic parameter for virus or bacterium cell classification.

From the analysis of dynamics changes of electric voltage $U$ between electrodes 1 and 2 in time from the initial state without virus or bacterium cells to the final state with the presence of these cells in the test tube we can write expression for relation $\left(Q_{v 1} / m_{v 1}\right)$,

$$
Q_{v 1} / m_{v 1}=d\left(d / \Delta t^{2}-g\right) / U_{0}
$$

where $Q_{v 1}$ is the electric charge of the unit virus or bacterium cell and $m_{v 1}$ is mass of the unit virus or bacterium cell, $\Delta t$ is time of electric voltage of capacitor changes from the its initial state to the its final state, which is measurable.

Hence, we can find expression for mass of the tested unit virus or bacterium cell $m_{v 1}$ :

$$
m_{v 1}=Q_{v 1} U_{0} / d\left(d / \Delta t^{2}-g\right)
$$

Values $m_{v 1}$ and $\left(Q_{v 1} / m_{v 1}\right)$ are characteristic parameter for virus or bacterium cell classification also.

The surface plasmon resonance (SPR) realization condition $^{5}$ is:

$$
\sqrt{\varepsilon_{p}} \cdot \sin \theta_{\text {res }}=\sqrt{\frac{\varepsilon_{g} \cdot \varepsilon_{1}}{\varepsilon_{g}+\varepsilon_{1}}},
$$

which allows to determine the permittivity of virus or bacterium cells substances $\varepsilon_{1}$, where $\theta_{\text {res }}$ - resonance angle of SPR, $\varepsilon_{g}$ - permittivity of graphene, $\varepsilon_{p}$ - permittivity of optical material under the upper graphene film electrode 1 . Parameter $\varepsilon_{1}$ is characteristic parameter for virus or bacterium cell classification also. 
The relation ${ }^{10}$ of $\varepsilon_{1}$ with thickness $d_{1}$ of virus cells layer on upper electrode 1 is

$$
\left(d-d_{1}\right)+\frac{\varepsilon_{2}}{\varepsilon_{1}} d_{1}=\frac{\varepsilon_{2}}{C_{3}} .
$$

Here $d$ is diameter of tested tube (or distance between electrodes), $\varepsilon_{2}$ is permittivity of substance between electrodes at the absence of virus or bacterium cells. The value $C_{3}$ is an effective capacity of test tube at the presence of virus or bacterium cells, which can be experimentally determined. So, we can find the characteristic parameters of virus or bacterium cells $\varepsilon_{1}$ and $d_{1}$ for virus or bacterium cell classification.

The frequency of plasmon waves $\omega_{p}$ is ${ }^{6}$ :

$$
\omega_{p}=e \sqrt{\frac{n}{m \cdot \varepsilon_{0}}},
$$

where $e$ - electric charge of electron, $m$ - mass of electron, $n$ - density of valence electrons in graphene.

By knowing the values of $\varepsilon_{1}, d_{1}, v_{v 1}, m_{v 1}, Q_{v 1}$ and $\left(Q_{v 1} / m_{v 1}\right)$ we can determine the type of the tested virus or bacterium cell.

Biosensors based on SPR phenomena and graphene have high sensitivity and good detection limit in $\mathrm{pg} / \mathrm{ml}$ of different viruses, bacteria, and other biological objects. This method may be applied for rapid in vitro and in vivo on-line detection. Such device is low-cost, simple and convenient. Such approach allows provide classification of different viruses and bacteria.

\section{BIOLOGICAL AGENTS DETECTION BASED ON GRAPHENE NANOTRANSISTOR}

Structure and properties of viruses. Size of most viruses is in the range from 10 to $300 \mathrm{~nm}$. On the average, viruses are 50 times smaller than bacteria. They can not be seen in the optical microscope, because their size is less than light wavelength 7.

The protein shell is called as capsid. The shell is often constructed from identical repeated subunits capsomere. Capsomere form structures that characterized with high symmetry. Additional lipoprotein envelope comes from the plasmatic membrane of a host cell and is found in relatively complex viruses (influenza virus, herpes virus). Fully formed infectious viral particle is called virion.

There are viruses that multiply in the cells of animals, others chose the plants, the third (called bacteriophages or phages) parasitize in the microbes. Although icosahedral form is found in viruses of all virus groups, there are still some differences. For example, virions with a head and tail appendix are characteristic for phages. Viruses with spiral and filament form usually parasitize on plants.

The structural features of infected cells are one of the factors that determine the form of virion. Since bacteria and plant cells in addition to the membrane of the cytoplasm are surrounded by a sufficiently dense and strong walls thus overcoming such wall is a serious problem for viruses. Many phages solve it by using a special device which resembles injector. Hence, there are their favorite form in which their tail appendix acts as a needle. Plant viruses to pass through the wall often with help of mechanical damage that is caused by insects or agricultural instrumentations. Through such cellular injuries into the host cells can penetrate viruses, which have not special gears for infection of intact cells.

Graphene based nanotransistor. Graphene with its two-dimensional nature has already found wide range of applications in the field of plasmonics, photonics and optoelectronics due to its unique and paramount characteristics as well as ease of compatibility and implementation on complementary metal-oxide-semiconductor (CMOS) technology. Furthermore, as 
graphene is atomically thin layer of carbon atoms, it is biocompatible and has large surface to volume ratio ${ }^{8,9}$ it can easily be employed for label free sensitive biosensors construction.

Graphene based electronic biosensors have significant advantages compared to optical, biochemical and biophysical methods in terms of high sensitivity, high spatial resolution for localized detection and ease of integration with CMOS technology. The architecture of biosensor based on field effect transistor (FET) geometry attracted a lot of attention since it provides label-free real time direct detection of interaction between target biological agents with FET surface in readable electrical signals.

In field effect transistors the current flow in channel between source and drain of the device. The conductivity of channel is modified between On and Off state by variation of gate electrode potential that is capacitively connected to it thought high-k $\mathrm{HfO}_{2}$ dielectric layer. In FET-based biosensors, graphene channel is in direct contact with the environment that provides better control over the surface charge variation due to adsorb biomolecules agents onto the graphene channel surface. Biosensor based on graphene field-effect transistor (FET) is controlled by the change in channel surface potential caused by molecules binding to graphene surface. When charged molecules such as biomolecules bind to channel surface of FET they can change the charge distribution along the graphene channel which leads to a change in the conductivity of FET channel as a function of applied gate bias of the device and the change of graphene channel conductivity is connected to specific diameter of adsorbed biomolecules to its surface ${ }^{10}$.

\section{REFERENCES}

[1] Hoaa X.D., Kirkb A.G., Tabriziana M., "Towards integrated and sensitive surface plasmon resonance biosensors:a review of recent progress”, Bios. Bioelectronics., 23, No.2, 151-160 (2007).

[2]. Homola J., "Surface Plasmon Resonance Sensors for Detection of Chemical and Biological Species", Chem. Rev., 108, 462493 (2008).

[3] Abbas A., Linman M.J. and Cheng Q. "New trends in instrumental design for surface plasmon resonance-based biosensors”, Biosens Bioelectron., 26(5), 1815-1824 (2011).

[4] Aznakayev E.G., Aznakayeva D.E., "Classification, Identification and Determination of Biological Agents with Graphene Nanosensor”, Proceedings of IEEE Conference MRRS-2014, Kiev, Ukraine, 107-110 (2014).

[5]. Maier S.A., Plasmonics: Fundamentals and Applications: Springer Publishers, Germany (2007).

[6]. Bonod N., Enoch S., Plasmonics: From Basics to Advanced Topics:Springer-Verlag Publishers, Berlin Heidelberg (2012).

[7] Aznakayev E.G. Biomedical Processes Modeling. Genomics: Osvita Ukraini Publishers, Kiev (2011).

[8] Wang Y., Li Z., Wang J., Li J. and Lin Y., “Graphene and graphene oxide: biofunctionalization and applications in biotechnology”, Trends in Biotechnology, 29(5), 205-212 (2011).

[9] Pumera M., “Graphene in biosensing”, Materials Today, 14(7), 308-315 (2011).

[10] Moraes A. C., Kubota L. T., "Recent Trends in Field-Effect Transistors-Based Immunosensors”, Chemosensors, 4(4), 20, 1-26 (2016). 